Revue des patrimoines

$20 \mid 2013$

Les patrimoines de la traite négrière et de l'esclavage

\title{
PREFACE. Mémoires et patrimoines vivants de la traite négrière et l'esclavage
}

\section{Françoise Vergès}

\section{OpenEdition}

\section{Journals}

Édition électronique

URL : http://journals.openedition.org/insitu/10265

DOI : $10.4000 /$ insitu. 10265

ISSN : $1630-7305$

\section{Éditeur}

Ministère de la culture

Référence électronique

Françoise Vergès, «PREFACE. Mémoires et patrimoines vivants de la traite négrière et l'esclavage », In Situ [En ligne], 20 | 2013, mis en ligne le 14 mars 2013, consulté le 23 avril 2019. URL : http:// journals.openedition.org/insitu/10265; DOI : 10.4000/insitu.10265

Ce document a été généré automatiquement le 23 avril 2019

\section{(c) $($ ) $(9)$}

In Situ Revues des patrimoines est mis à disposition selon les termes de la licence Creative Commons Attribution - Pas d'Utilisation Commerciale - Pas de Modification 4.0 International. 


\title{
PREFACE. Mémoires et patrimoines vivants de la traite négrière et l'esclavage
}

\author{
Françoise Vergès
}

«Mais les objets avaient voyagé, apportés par
d'innombrables trocs
et une infinité de traversées.
Ils étaient là, énigmatiques et solennels,
dans tout le système de leur facture ».
Alejo Carpentier, Le Siècle des Lumières (1962)

1 La loi votée le 10 mai 2001, dite Loi Taubira, reconnaissant la traite négrière et l'esclavage comme «crime contre l'humanité » a donné une nouvelle impulsion à la recherche sur plusieurs siècles d'histoire qui ont marqué l'histoire de l'Europe, de la France, de l'Afrique, des Amériques, des Caraïbes et des îles de l'océan Indien. Ces dernières années ont vu apparaître de nouvelles lectures et de nouveaux regards: itinéraires singuliers, histoires de famille, registres d'état-civil, registres de plantations, patrimoine mobilier, iconographie... sont examinés sous de nouveaux angles. Les patrimoines, matériels ou immatériels, sont réexaminés, relus, redécouverts. Un croisement des sources, une approche transversale ont ouvert de nouveaux champs d'investigation.

\section{Une approche transversale, de nouveaux champs d'étude}

2 L'histoire de la traite négrière, de l'esclavage et leur abolition nous offre de nombreuses sources de réflexion, sur le travail, le droit, la liberté, la dignité, les mentalités, les arts... Or, malgré des travaux importants, un effort reste nécessaire pour que soit reconnu le rôle central que cette histoire a joué dans l'émergence de la modernité, dans l'histoire de l'Europe et du monde, dans l'image que l'Europe s'est donnée d'elle-même, dans la construction de l'Afrique comme continent de la misère, dans la création de mondes 
nouveaux, les mondes dits « créoles » et la singularité de leur culture, de leurs langues, et de leurs savoirs. L'émergence des notions de liberté, de dignité, de fraternité, et des droits imprescriptibles de la personne humaine sont indissociables de cette histoire. Pour penser la liberté, les philosophes et les juristes lui ont opposé la servitude, pour penser la dignité, la déshumanisation. La formule inventée en 1787 par Josiah Wedgwood, abolitionniste anglais, Am I not a man and a brother ?, qui fut reprise par les abolitionnistes français ( Ne suis-je pas un homme et donc ton frère?») et orna tabatières, coupes, assiettes et badges, montre à quel point l'esclavage colonial posa cette question à l'Europe : qui faisait partie de l'humanité ? Si tous les hommes (sic) étaient frères, s'ils partageaient leur humanité et ce quelle que soit leur religion, leur pays, leur couleur de peau, l'esclavage était injustifiable. Ce principe fonda la Déclaration des droits de l'Homme et du Citoyen. Cette affirmation d'une humanité commune fut au cœur des luttes des esclaves. Leurs voix ont pour une grande part été effacées dans le monde colonial français, mais en cherchant dans les archives, en menant des fouilles archéologiques, en collectant les mémoires orales et les expressions vernaculaires, nous pouvons redécouvrir ce patrimoine. Ce sont souvent des bribes, des traces, des fragments, mais ils permettent d'évoquer la singularité d'une vie. L'histoire de Furcy, esclave réunionnais, qui lutta vingt-sept ans pour obtenir une liberté qui lui était due a été retrouvée grâce à un long et patient travail dans les archives ${ }^{1}$. Mais l'esclave reste une figure multiforme et protéiforme, si diverse dans le temps et l'espace qu'il semble pratiquement impossible de la saisir sous un seul vocable. C'est une figure qui parle dans un grand nombre de langues, dont l'expérience intime est chaque fois singulière, mais qui rejoint une expérience collective. C'est une expérience si dispersée qu'elle ouvre une multiplicité de possibles, où il faut tenir compte des diversités de statuts, des itinéraires spécifiques, du contexte culturel, géographique, social, légal et géopolitique. C'est un monde mouvant. Cette difficulté vaut pour toutes les catégories, dominés comme dominants, mais celle de l'esclave reste la plus opaque et la plus polysémique, ce qui explique paradoxalement qu'elle soit une référence si fréquente.

3 Cependant, il faut rappeler combien la lutte incessante des esclaves pour leur liberté a contribué à l'extension des idéaux de la démocratie, à l'universalité des droits humains et que le mouvement anti-esclavagiste fut l'un des premiers grands mouvements internationaux pour les droits humains. Une nouvelle génération de chercheurs émerge, attentive au croisement des sources, à l'histoire culturelle, du genre, du patrimoine industriel, des réseaux d'intérêts...

\section{Le Comité pour la mémoire et l'histoire de l'esclavage}

4 Le Comité pour la mémoire et l'histoire de l'esclavage (CPMHE) ne peut que se féliciter de ce renouveau. Issu de la loi du 10 mai 2001, le CPMHE s'est donné pour objectif principal de restituer cette histoire à tous les citoyens. Pour ses membres, il est inconcevable de transformer ces siècles en une parenthèse regrettable. Pour rappel, la loi est l'aboutissement d'années de mobilisation de la part d'associations, de chercheurs et d'élus. En 1998, lors du $150^{\mathrm{e}}$ anniversaire de l'abolition de l'esclavage dans les colonies françaises, nombreux sont ceux qui s'émeuvent de l'orientation donnée par le gouvernement à cette célébration: encore trop de place accordée aux abolitionnistes français, encore un effacement des esclaves, de ces femmes, ces enfants, ces hommes, capturés et mis en esclavage, ou nés dans la servitude et qui furent les ancêtres de tant de 
citoyens. Le 23 mai 1998 a lieu à Paris une manifestation qui s'oppose à cette approche et affirme la nécessité de se souvenir des esclaves. Dès le milieu de 1998, des propositions de loi sont déposées à l'Assemblée nationale tendant à la reconnaissance de la traite négrière et de l'esclavage comme "crime contre l'humanité ». Il y en aura plusieurs avant que le gouvernement retienne celle qui sera défendue par la députée Christiane Taubira. L'étude des débats de la commission montre qu'il y a peu de divergences, le gouvernement socialiste a écarté toute mesure de réparation, les seules qui faisaient controverse. Certains députés jugent que la spécification de l'esclavage comme «crime » figure déjà dans le Code Pénal mais aucun ne s'oppose fermement à l'adoption de la loi, votée à l'unanimité en deuxième lecture au Sénat le 10 mai 2001. Elle est inscrite au Journal Officiel le 21 mai 2001.

5 La loi prévoit l'installation d'un comité de douze personnalités, issues des associations, du monde de la recherche et de l'Outre-mer. Installé une première fois en 2002, le Comité pour la mémoire de l'esclavage (CPME) est mis en place définitivement en janvier 2004 et ce pour 5 ans. Ses membres, tous bénévoles, ont pour mission de faire des propositions au gouvernement: proposer une date nationale des mémoires de la traite négrière, de l'esclavage et de leur abolition, et des mesures dans l'Éducation nationale, la recherche et la culture. Il rend son premier rapport en 2005 et propose comme Journée nationale des mémoires de la traite négrière, de l'esclavage et de leur abolition le 10 mai, date du vote de la loi, et fait des recommandations pour les programmes et les manuels scolaires après en avoir fait un bilan, comme des recommandations dans la recherche et la culture, notamment la création d'un Mémorial/centre de ressources/centre culturel sur les traites et les esclavages ${ }^{2}$. Dès son installation, le Comité prend contact avec de nombreuses institutions, dont la Direction des musées de France et les Archives de France. Toutes les deux se montrent très ouvertes à une collaboration. En 2006, est lancé avec la Direction des musées de France un inventaire des objets relatifs à la traite négrière, l'esclavage et leur abolition. L'idée germe alors d'une grande exposition s'appuyant sur les collections des patrimoines. En 2007, les Archives de France publient le Guide des sources de la traite négrière, de l'esclavage et de leurs abolitions, sous la direction de Claire Sibille (Paris : Direction des Archives de France). En 2006, la première Journée nationale des mémoires de la traite négrière, de l'esclavage et de leur abolition est célébrée officiellement. Un monument est inauguré dans les Jardins du Luxembourg en 2007. En 2009, quand le gouvernement choisit de reconduire l'action du Comité qui devient alors « Comité pour la mémoire et l'histoire de l'esclavage", ce dernier choisit comme axes de son action, l'éducation et la médiation, c'est-à-dire de mettre l'accent sur les moyens de faciliter l'accès de cette histoire au plus grand nombre, et ce par les moyens les plus divers: cinéma, théâtre, littérature, expositions, ouvrages scientifiques, colloques... Il faut imaginer à terme l'existence d'un lieu tangible - Historial, Mémorial, Centre de documentation et d'exposition...- où trouver de la documentation et des réponses aux questions qui se posent.

6 En effet, le CPMHE estime que si la recherche a trouvé un nouveau souffle, éducation et médiation citoyenne souffrent de retard. Or, ses membres estiment que la connaissance de cette histoire constitue un acte citoyen, qui nous fait tous réfléchir sur les formes d'exploitation, la servitude, la déshumanisation, la résistance à l'oppression et la construction du bien commun. Il ne s'agit ni de repentance, ni de procès révisionniste mais d'inscrire ces siècles d'histoire dans une approche croisée. L'esclavage est contemporain de la modernité, de la Déclaration des droits de l'Homme et du Citoyen, de 
la fin de la monarchie, des révolutions des Lumières... Les traites et l'esclavage transforment profondément la cartographie du monde, globalisent des économies, affectent le droit, la philosophie, les arts et mettent en contact des cultures, des langues, des savoirs et des croyances. Et l'anti-esclavagisme constitue toujours une source d'inspiration dans la lutte pour les droits humains, la dignité et la liberté.

\section{Héritages complexes et multiples}

7 Les héritages de l'esclavage sont complexes et multiples: expérience de l'exil de la déportation, de la déshumanisation et création de nouvelles cultures, croyances et savoirs. Les sociétés et cultures créoles en sont des témoins. Il faut chaque fois prendre en compte les transformations auxquelles est soumis le système esclavagiste, fruits des rivalités entre puissances européennes, entre royaumes africains négriers, des conséquences de révoltes, d'insurrections, de la Révolution haïtienne, des reculs et des avancées du mouvement abolitionniste occidental. Le monde esclavagiste est un monde mouvant et il est réducteur d'en faire un récit linéaire.

8 Si la condamnation de l'esclavage ne pose plus problème, la compréhension du phénomène reste difficile. Comment expliquer le consentement d'un monde au commerce d'êtres humains et à leur mise en esclavage alors que ce monde affirme au même moment la doctrine des droits naturels, la force de l'approche scientifique, et l'importance de la Raison? Comment expliquer que cela dure des siècles? Pourquoi les révoltes d'esclaves n'ont-elles pas été plus souvent couronnées de succès? Pourquoi des Africains ont-ils accepté de participer au commerce de femmes et d'hommes qui étaient leurs voisins? Qui a profité de ce commerce ? Ces questions soulignent l'importance de la diffusion du savoir et des connaissances pour dépasser les clichés, questionner les demi vérités, entrer dans la complexité des faits. Ce n'est pas l'histoire du bien et du mal, mais de la mise en place de vastes réseaux d'intérêts divers et convergents. Rendre accessible les sources patrimoniales participe activement à ce travail de restitution pour rendre compréhensible un "crime contre l'humanité ». En France, au CPMHE, nous avons souvent constaté une attention réelle aux formes contemporaines de l'esclavage mais une plus grande difficulté à comprendre les héritages de l'esclavage colonial. Nous avons perçu rarement de l'hostilité, parfois de la gêne - qui est responsable ? Pourquoi cela dure si longtemps? Par contre, nous avons noté un refus chez certains élus et historiens d'accepter de comprendre ce qu'ont représenté traite et esclavage pour le monde, ou d'admettre que le racisme anti-Noir y trouve ses origines. Il y a comme une difficulté à admettre le poids de cette histoire et à analyser le racisme anti-Noir qui trouve son origine dans la traite négrière et l'esclavage colonial.

Dix ans après l'adoption de la loi, quel est le bilan? La France dispose aujourd'hui de plusieurs dates commémorant cette histoire, expression de sa durée et de sa configuration multiple. Le 10 mai est la Journée nationale des mémoires de la traite négrière, de l'esclavage et de leurs abolitions (décret 2006). Dans l'hexagone, le 23 mai est une journée dédiée à la mémoire des victimes de l'esclavage, en référence à la manifestation de 1998. Depuis 1983, un décret a institué un jour férié de célébration de l'abolition de l'esclavage: le 27 avril à Mayotte; le 22 mai en Martinique; le 27 mai en Guadeloupe ; le 10 juin en Guyane ; le 20 décembre à La Réunion.

De nombreux progrès ont été accomplis dans les domaines de l'enseignement, de la recherche et de la valorisation des patrimoines. Un Centre international de recherche sur 
les esclavages a vu le jour au CNRS, de nombreux ouvrages sont parus, le nombre des thèses a augmenté considérablement et les thèmes de recherche se sont diversifiés, des créations culturelles et artistiques ont pris traite négrière et esclavage comme thèmes. Les Archives nationales comme certaines archives départementales ont engagé des actions de numérisation et de médiation, des salles ont été ouvertes au musée des ducs de Bretagne et au musée d'Aquitaine.

11 Le CPMHE ne peut que se féliciter de ce renouveau. Le programme triennal lancé par la Direction générale des patrimoines va contribuer à élargir les champs d'étude. Le CPMHE estime pourtant qu'il reste encore à faire en particulier dans le domaine de l'enseignement et de la médiation. Trop de méprise et d'incompréhension demeurent. Trop de Français connaissent encore mal cette histoire. Il est inconcevable que les apports des esclaves et de leurs descendants soient ignorés ou marginalisés. Il est inacceptable que des siècles qui ont vu des êtres humains mis en esclavage, privés de droits civiques, de patrimoine, et de patronyme, soumis au fouet et aux fers, et qui ont contribué à la richesse économique de la France, soient oubliés.

Le travail que j'ai accompli depuis 2004 au Comité et pendant six ans, de 2003 à 2007, à la tête de l'équipe de conception et de préfiguration de la Maison des civilisations et de l'unité réunionnaise ${ }^{3}$, m'a menée à revisiter le statut de l'objet dans les patrimoines de la traite négrière, de l'esclavage et de leur abolition, ce que j'appelle «l'objet de l'immatériel », ainsi que la question du "genre » de l'esclavage, la nécessité de retracer de nouvelles cartographies et de s'interroger sur le rôle de la mémoire comme pratique sociale.

\section{L'objet de l'immatériel}

13 Partant de la notion de «biographie de l'objet» comme l'a définie Igor Kopytoff ${ }^{4}$, je considère son itinéraire, ses usages, cette part immatérielle que lui donne sa vie sociale et culturelle. Pour Kopytoff, quand un objet, une idée, une pratique, et une personne entrent en contact, chacun exerce une pression sur l'autre. La biographie de l'objet cherche à mettre en lumière l'objet comme " agent » à travers sa " vie ", passant de main en main, il transforme celui ou celle qui l'utilise et se transforme. L'objet entre en relation dynamique avec la vie humaine et cette relation est elle-même dans une relation dynamique avec le contexte social et culturel. L'objet n'est pas passif et sa relation avec les personnes appartient au monde de la sensation et de l'émotion. Cette approche de la culture matérielle n'est bien sûr pas spécifique à l'esclavage et cette méthodologie est aujourd'hui assez répandue dans l'étude du monde matériel. Pour la traite et l'esclavage, il s'agit de l'appliquer à l'objet immatériel : comment circulent et se transforment les langues, les rites, les musiques, les savoirs, les croyances? Comment retracer la «biographie » d'un objet immatériel?

Tout objet raconte une histoire, a une biographie, un "passeport» sur lequel s'accumuleraient les tampons qui racontent le passage de frontières. Passant de main en main, de marché en marché, de port en port, l'objet voyage, s'installe dans une maison puis dans une autre pour finir parfois au bord du chemin jeté et oublié, emportant avec lui des souvenirs, des sentiments.

15 L'objet n'est pas seulement "matériel », objet fabriqué, échangé, vendu. Il porte toujours les traces immatérielles de ces moments de fabrication et d'échange. Sa vie constitue un fait 
humain et social. Tout cela est très présent dans la traite et l'esclavage : ces circulations de croyances, de langues, de savoirs, de musique, de pratiques culinaires, de technologies. Tracer leurs routes, c'est redonner à ces objets leur dimension humaine et sociale.

Les artefacts manquent qui témoigneraient de la complexité des vies des pauvres et des opprimés. Il existe peu d'objets pour raconter l'histoire de la vie des premiers colons, des esclaves, des travailleurs engagés, et des migrants. En effet, avec quels objets raconter l'espoir d'un jeune colon ayant quitté la misère des campagnes françaises, pour faire fortune dans les colonies? Avec quels objets raconter sa rencontre avec une île tropicale, avec les esclaves malgaches, africains, indiens avec le système économique et culturel colonial ? Quels furent ses rêves, ses révoltes, ses joies? Avec quels objets raconter la détresse d'un jeune malgache, d'un jeune africain déporté dans une colonie, son arrivée dans un monde inconnu, sa première rencontre avec le système esclavagiste de plantation? Avec quels objets raconter sa découverte du Créole, des rites de la nuit, du marronnage, de la lutte et des résistances?

Les cultures issues de l'esclavage se sont traduites surtout par des créations où l'immatériel domine. Elle ne s'est pas inscrite à travers des monuments, des palais, des forteresses... Les grandes maisons créoles sont des maisons de maitre et racontent l'histoire d'un très petit nombre. C'est à travers la musique, la cuisine, les rites, les repas collectifs, le pique nique, que ces créations s'expriment. La dimension matérielle s'efface en grande partie derrière le moment immatériel, celui où chacun se retrouve dans le collectif, moments communs où la conversation, l'échange, le rire, les pleurs construisent ce qui va demeurer dans le souvenir, les traces d'un moment partagé.

Dans un texte paru en 2004, Carpanin Marimoutou, poète, écrivain et professeur à l'Université de La Réunion et moi-même, avions déjà cherché à expliciter cette dimension dans le monde colonial. Nous écrivions alors à propos du Créole réunionnais :

«Paroles de maîtres à esclaves, d'esclaves à maîtres, d'esclaves à esclaves, de maîtres à engagés, d'engagés à maîtres, d'engagés à engagés, de libres à libres. Les discours et les savoirs sur le monde, versés au langage sous forme de sens à construire, sont produits à partir de la perception et de l'expérience du lieu et des rapports de production sur le lieu. Mais cette langue porte nécessairement en elle, dans l'hétérogénéité même qui préside à son élaboration, la marque des langues, des rêves, des imaginaires qui ont présidé à sa naissance; versés en inconscience, souterrains, cryptiques. Mais cela resurgit, d'une façon ou d'une autre, dans la parole quotidienne de l'échange, dans la parole poétique, dans les textes des ségas et des maloyas, dans les proverbes, les jeux de mots, les devinettes. L'hétérogénéité resurgit, mais transformé par les rencontres d'imaginaires qui produisent les imaginaires du lieu; cela resurgit dans les croisements et les appropriations. Une légende, Granmèr Kal, se construit en amalgamant des mythes de l'Inde, de Madagascar, de l'Afrique à une mémoire populaire des traditions orales réunionnaises. Cette mémoire est liée à l'appréhension que les esclaves ont du maître et de ses pouvoirs, à une perception spécifique du surnaturel. Cela resurgit dans un maloya de Firmin Viry où l'héroïne d'une épopée indienne, Sita dans le Ramayana, - transformée en une ouvrière des plantations, rencontre une ancienne romance française. Cela resurgit dans les spectacles de rues, qui mêlent l'espace profane à l'espace du sacré, comme dans le cas du jako qui transporte dans sa danse et sa gestuelle des mythes et des pratiques réinterprétées de l'Inde dravidienne et du Mozambique. Cela resurgit dans le Narlgon - théâtre tamoul ou malbar - où ce qui relevait du rituel dans l'espace originel devient spectacle théâtral à la place du Terukkutu versé en inconscience. Cela resurgit sans doute à l'insu des énonciateurs eux-mêmes, qui ont mis les origines entre parenthèses, mais cela est là, toujours présent et immédiatement réutilisable. Le maloya met en scène cet espace de 
créolisation india-océanes, l'espace commun d'un ethos réunionnais. Le texte chanté du maloya n'acquiert signification et valeur qu'en contexte festif, cérémoniel, que dans l'interaction interne (chanteur/chœur) et externe (troupe/public participant). Le texte du maloya, souvent improvisé à partir d'un fonds dont l'origine est difficilement déterminable, est variable à l'infini, en fonction des conditions d'énonciation, de la participation du public, de l'état d'esprit du chanteur, de la forme du chœur; bref, le maloya est une performance. À la fois pratique sociale et pratique discursive, le maloya est aussi à lire comme texte avec ses logiques internes, ses déconstructions/reconstructions de la parole collective reçue, ses resémantisations, et comme le texte particulier d'un énonciateur particulier.

C'est la communauté en représentation langagière, discursive, sociale. Ce qui était marginal devient ainsi central. Le maloya brouille et multiplie les positions et les identifications ${ }^{5}$."

Les archives recèlent des trésors sur ces circulations de l'immatériel, les itinéraires, les échanges. Pier Larson dans sa magnifique étude, Ocean of Letters. Language and Créolization in an Indian Ocean Diaspora, explore le rôle des langues africaines dans l'impérialisme et la créolisation à partir de lettres échangées, d'archives de procès, de témoignages de missionnaires ${ }^{6}$. En relisant ces archives, il démontre la centralité de la langue malgache dans les colonies esclavagistes européennes de l'océan Indien. Il analyse parallèlement comment, à l'époque de l'esclavage, linguistique et pensée racialisante sont imbriquées. Ces observations l'amènent à critiquer la manière dont la créolisation a été pensée comme effacement des langues d'origine. La focalisation sur l'hybridité culturelle et linguistique dans les sociétés esclavagistes, écrit-il, marginalise le processus simultané d'extinction et d'utilisation de langues africaines. Il conclut sur l'expérience cosmopolite d'esclaves, qui font des va et vient entre territoires, qui circulent entre langues et cultures. Quelle leçon tirer de cette étude ? Elle souligne l'importance des regards croisés, l'attention à porter à la langue, et une méfiance envers toute méthodologie qui cherche à opérer une compensation, ici privilégier l'hybridité alors qu'il s'agirait plutôt d'étudier l'interaction entre le vernaculaire et la créolisation. Larson montre l'importance de régionaliser l'étude de la traite et de l'esclavage puis d'entreprendre une comparaison, car l'importance numérique des Malgaches parmi les esclaves des colonies de l'Océan Indien (jusqu'à $40 \%$ des effectifs) entraîne une créolisation différente de celle des Caraïbes.

20 L'archive immatérielle, la tradition orale, recèle aussi des informations importantes. En Afrique, la tradition orale sur la "période des chevauchées permanentes » a gardé en mémoire, « l'ampleur des ravages opérés par certaines maladies », le développement de l'alcoolisme, la régression de l'agriculture, la prostitution, les disettes et les famines ${ }^{7}$. Une formule en wolof exprimait le principe selon lequel la force fait la loi : «Le statut de prince ne peut s'acquérir et se conserver que par la possession de montagnes de fusils ${ }^{8}$.» La traite coloniale était utilisée comme menace: "Tu as intérêt à changer, sinon tu t'exposes à être vendu au Rio Pongo ! » disait le maître à l'esclave'

21 Je pourrai parler du maloya, du gwo ka, des arts de la fuite, des savoirs médicaux, des recettes de cuisine..., tous ces exemples de culture immatérielle, avec leur dynamique propre, leurs reconfigurations, leurs réinventions, qui démontrent que l'objet de l'immatériel a toute sa place dans les patrimoines de la traite négrière, de l'esclavage et de leur abolition. La peur d'encourager le fantasme a amené à se focaliser sur l'archive écrite, qui, je le répète, n'a toujours pas livré tous ses trésors. Mais l'archive orale et les patrimoines immatériels doivent être croisés avec le document écrit. L'archive orale est une réalité sociale et culturelle, en tant que telle, elle est objet d'étude. 
Cette présence de l'immatériel comme archive de l'esclavage nourrit la réflexion sur une muséographie de l'esclavage qui se fonderait sur l'expérience des esclaves. En partant d'une absence d'objets matériels - car les chaînes, les fouets, les actes notariés, les registres, les rapports de police ou de tribunal, disent peu de ce qu'un(e) esclave rêvait, créait, de sa vie intime - tomber amoureux, ressentir du désespoir, pleurer la mort d'un proche... - ou de son expérience de travail. Pour ne pas réduire la personne mise en esclavage au statut d'esclave, qui lui fut imposé, il faut, sans tomber dans le romantisme et l'idéalisation, faire l'effort d'évoquer cette part d'humanité.

\section{Le « genre » de l'esclavage}

Les conceptions que se font les sociétés européennes et africaines, puis les sociétés esclavagistes et les communautés d'esclaves des notions de masculinité et de féminité influencent la répartition des rôles et des processus d'identification homme/femme. Le corps esclave, masculin ou féminin, est cependant soumis à des violences semblables. Il est entièrement dénudé lors de l'embarquement, soumis aux gestes brutaux des acheteurs, des marins, des propriétaires, fouetté de la même manière. Le corps esclave est à la fois sexualisé (les femmes occupent des fonctions "féminines", sages-femmes, cuisinières, domestiques, bonnes d'enfants) et désexualisé (les femmes travaillent aussi dur que les hommes).

Sur le continent, des femmes esclaves accompagnent les convois d'esclaves, employées à transformer en farine le millet ou le sorgho et à le cuire pour nourrir le convoi. Ce sont en majorité des enfants, des femmes et des personnes âgées qui sont dans les ports négriers africains, chargés de transformer le manioc (qui a été rapporté des Amériques) en farine, car cette dernière, qui sert à nourrir les esclaves transportés aux colonies, est facile à transporter sur les bateaux négriers. Sur ces bateaux, les femmes remplissent parfois la même fonction ${ }^{10}$. Là ou la prédation fait la distinction de genre la plus "marquante", c'est en utilisant le viol comme arme de guerre contre les femmes.

Le déséquilibre de la traite qui affecte, sur le continent africain, les « rapports de genre et de génération des populations puisqu'elle visait de manière privilégiée les jeunes adultes (mâles ou femelles selon les cas) les plus vigoureux et les plus féconds ${ }^{11}$ ", affecte aussi des colonies esclavagistes.

L'étude de la situation des femmes esclaves montre des variations d'une colonie à l'autre, d'une plantation à l'autre. Ce que je veux signaler ici c'est que l'histoire fortement sexuée de la traite et de l'esclavage montre la nécessité d'affiner les études sur le genre, la prédation, et l'esclavage ${ }^{12}$. Il me parait intéressant de clarifier comment les notions de masculinité et de féminité se sont construites dans un réseau de significations où se jouent des schémas hérités (réels ou réinventés) de l'esclavage, du statut colonial et des politiques assimilationnistes.

\section{Nouvelles cartographies}

Les cartes déterminent la manière dont nous pensons le monde. "Créant » des mondes, elles se lisent comme un texte qui nous aide à les comprendre. Les géographes ont montré la complicité de la cartographie avec la conquête coloniale, comment l'iconographie des empires coloniaux a aidé les peuples colonisateurs à « voir» le monde depuis l'Europe. 
Avec les indépendances, les cartes nationales ont tracé de nouvelles frontières. Retracer les cartes croisées des traites et des esclavages fait apparaitre une autre cartographie de la modernité et des échanges sud-sud.

Cette cartographie - des sentiers de marronnage, des chemins empruntés pour les rites, du chemin du travail, du repos, des résistances, des renoncements, des luttes, des défaites...- dessine une histoire des territoires singuliers, des sans noms, des sans part. La carte des marchés - des esclaves comme des produits - et celles des ports négriers font apparaitre d'autres imaginaires, d'autres buts et intérêts que ceux tracés par la carte des histoires nationales.

29 À ces cartes s'ajoutent celles des résistances : villes et villages refuges sur le continent africain, révoltes sur les bateaux négriers, territoires des marrons dans les colonies, routes des diasporas, propagation des idées, des rituels, des savoirs. Si traite et esclavage dessinent une cartographie de la violence, celle des résistances dessine une contrecartographie culturelle et politique.

La route des esclaves est celle ouverte par les négriers, mais quelle est réellement celle des esclaves ? Comment imaginent-ils le monde à partir des bouleversements dont ils font l'expérience, tant pour ceux qui font le voyage dans la cale négrière que pour ceux qui naissent sur les plantations? En 2002, je me trouve à Inhambane, ville de la cote du Mozambique d'où partaient les esclaves pour les îles Mascareignes, entre autres destinations. Les esclaves, dit la mémoire locale, étaient embarqués de nuit, les yeux bandés. Ils « voyaient » de nouveau, une fois la côte éloignée. Ils découvraient ensuite un monde qui allait devenir le leur, une colonie européenne où tout leur était étranger. Quelle carte ont-ils construite? Sans doute pas celle que nous appelons "route de l'esclave ».

31 Autre exemple, celui des témoignages d'esclaves nés sur des plantations, qui parlent des chemins empruntés la nuit pour rejoindre un ami, une amante, de lieux de culte et de prière, de lieux cachés. Dans son autobiographie, Frederick Douglass raconte que sa mère, alors qu'il était enfant, parcourait en courant des dizaines de kilomètres pour le retrouver la nuit et repartir dans l'autre sens avant le jour. C'est une autre organisation spatiale que celle que nous fait découvrir la visite classique d'une plantation.

Mais plus encore, réinscrire l'esclavage colonial dans le champ du politique et de l'économie, c'est réinscrire l'Europe et ses colonies dans une histoire mondiale. L'Europe devient une des régions du monde et les colonies sont resituées à la fois dans leur région géographique et historique et dans les routes régionales d'échanges et de circulation d'hommes et d'idées. Les colonies ne sont plus des territoires dont l'histoire est exclusivement dictée par le lien à la métropole coloniale. Elles ont leur propre dynamique et sont au diapason des transformations des pays et des continents voisins.

Les Antilles françaises sont appréhendées dans leur voisinage avec le continent américain et l'archipel des Caraïbes ; la Guyane est réinscrite sur le continent sud-américain avec ses migrations, ses luttes et ses imaginaires ; La Réunion, dans l'Océan Indien, est un espace millénaire d'échanges, de rencontres et de conflits sud-sud, sur un axe Afrique-Asie.

Ce travail de décentrement du regard est un travail critique, qui ne vise pas à la construction d'une innocence perdue, mais à la relecture d'une histoire et d'une géographie moins axées sur la relation à l'hexagone. Ce décentrement redonne son rôle à la dynamique locale et régionale, aux mentalités, à une sociologie du politique. Ainsi, les expressions « indigènes » de racisme (propres au territoire colonial) ne sont pas ignorées, 
comme la paranoïa insulaire, le repli communautaire, l'instrumentalisation de certaines traditions, l'effet de la mondialisation sur les identités, l'angoisse devant un futur incertain. Les niveaux de signification se croisent, les cartes se complexifient, espace et temps de l'histoire s'étendent au-delà de l'espace étroit « métropole/colonie ». Cet axe ne peut entièrement expliquer comment ces sociétés se sont formées, comment des identifications se construisent, comment des traditions sont réinventées, des liens fantasmés, comment des «ethnicisations » de la mémoire voient le jour, comment les populations choisissent entre des héritages, comment elles les manipulent, les instrumentalisent et dans quel but.

L'esclavage interroge ainsi un récit eurocentré qui, comme l'écrit Frantz Fanon, par un « mécanisme de pensée somme toute assez banal, en arrive à ne plus pouvoir imaginer un temps se faisant sans lui $^{13} »$. Le décentrement bouleverse une notion hégémonique du temps ("grandes découvertes", colonisation...) et introduit des temporalités hybrides. Ce que l'anthropologue Jack Goody a appelé le «vol de l'histoire » opéré par l'Europe est analysé, et l'histoire européenne est croisée avec l'histoire globale, soumise à des accidents, des ruptures, des échanges ${ }^{14}$. «Conceptualiser et présenter le passé où l'on part d'événements qui se sont produits à l'échelle provinciale de l'Europe - occidentale le plus souvent - pour les imposer au reste du monde ${ }^{15} »$ construit des points aveugles dit Goody. C'est là mais on ne le voit pas.

En Afrique, cette relecture de la cartographie interroge le récit historiographique de compensation de mouvements anticoloniaux, récit qui, comme l'écrit Ibrahima Thioub, a " produit une histoire glorieuse de l'Afrique procédant par un gommage plus ou moins systématique de tout ce qui pouvait donner crédit à l'absence d'une historicité propre à l'Afrique ou à l'arriération de ces société » et la fiction qui veut que l'esclave fasse « partie de la famille ", cache la réalité de l'origine servile, stigmate qui le marque à vie et détermine ses positions sociales ${ }^{16}$. Cette relecture réinscrit l'Afrique dans les mondialisations et leurs régionalisations successives ${ }^{17}$, mettant au jour les relations intracontinentales, les routes sud-sud, les circulations d'idées ou de systèmes de croyance. Elle n'est pas déterminée par une relation exclusive à l'Europe. Finalement, cette relecture permet de mieux comprendre des phénomènes dans leur longue durée :

«Vous ne comprendrez pas que ce qui permet à ces rapports centre-périphéries d'avoir un impact aussi négatif sur les sociétés africaines. Vous ne verrez pas que c'est l'architecture sociale et politique de ces sociétés qui permet à ce facteur externe d'être recyclé par des groupes qui servent d'intermédiaires pour assoir leur domination. Donc, il faut redonner au sujet africain son statut de sujet historique et non pas d'objet ou simplement de victime ${ }^{18} »$.

\section{La mémoire comme pratique sociale}

Le long silence, suivi d'un mouvement de réappropriation, d'affirmation, n'a pas aidé à accepter le lacunaire, la complexité, le flou, le manque de certitudes. On a beaucoup glosé sur l'opposition entre mémoire et histoire, sur le fait que l'historien serait empêché de faire son travail de recherche par l'émergence de mémoires dites "militantes". L'écriture de l'histoire n'est pourtant pas une opération neutre. Chaque époque jette un nouveau regard sur le passé et nous relisons les archives à la lumière de ces nouvelles questions. Le chercheur vit dans un contexte social et culturel et n'est pas à l'abri de sa propre subjectivité ou du poids de son environnement. Témoignages et études sur l'évolution des sciences sociales qui mettent cet aspect en lumière abondent. Point n'est 
besoin de le déplorer. C'est un fait social. L'instrumentalisation et la manipulation des faits pour servir des intérêts particuliers ne sont pas toujours étrangers au désir de réviser l'histoire. Les mémoires de la traite négrière, de l'esclavage et de leur abolition constituent, de nos jours, une archive vivante. Cela peut étonner au premier abord: l'esclavage n'a-t-il pas été aboli en 1848 ? Mais ce serait oublier que la mémoire agit comme pratique sociale. Dans son ouvrage, Le Patrimoine en question, Françoise Choay interroge l'approche eurocentrée du patrimoine, devenu, écrit-elle, un mot clé de notre société mondialisée. J'ajouterai à cette critique de l'eurocentrisme, la critique d'une approche servant un récit nationaliste ou communautariste qui vise à effacer la complexité, les phénomènes d'hybridation, de perte, d'emprunt. Dans son intervention à un colloque à l'île Maurice en avril 2011, le Professeur Benigna Zimba de l'université de Maputo revenait sur les patrimoines de la traite dans un pays africain : elle parlait de la nécessité d'inclure les mémoires des marchands négriers locaux. En Guyane, les communautés Bushinenge (descendants d'esclaves marrons ayant fui le Surinam et s'établissant dans les forêts de ce qui deviendra la Guyane française) et Amérindiennes réclament la prise en compte de leurs mémoires singulières et questionnent l'hégémonie de la mémoire créole des descendants d'esclaves. La consommation mercantile du patrimoine, son exploitation touristique, mettent aussi en jeu des intérêts parfois divergents. Dans sa thèse sur la patrimonialisation de la montagne du Morne à l'île Maurice, Sandra Carmignano analyse ces divergences d'intérêts, l'interaction entre l'État, les associations, les chercheurs, et des organisations internationales comme l'UNESCO. La multiplication de stèles et de monuments cherche à combler un vide - l'absence d'inscription de cette histoire dans l'espace public - mais ces gestes ne visent pas une éducation citoyenne. Qui s'arrête devant une stèle ou un monument et prend le temps de comprendre? Quel message les monuments peuvent-ils faire passer s'ils ne sont pas accompagnés d'un programme éducatif qui les fait vivre?

Un seul événement, l'esclavage, a condensé des mémoires distinctes et entremêlées, contradictoires et collectives. La reconnaissance de ce pluralisme ouvre des espaces fructueux de recherche, au-delà des identités figées et territorialisées.

Il existe dans le monde contemporain une présence sociale et culturelle des patrimoines de la traite négrière, de l'esclavage et de leur abolition. À travers ces patrimoines, les vies des esclaves sont évoquées dans leur richesse, leur complexité. Ce ne sont plus des personnes "sans histoire " mais des êtres humains inscrits dans le monde par leurs créations, leurs paroles, leurs luttes et leurs chants. Les patrimoines vivants de cette longue histoire rendent hommage à ceux, pour reprendre les mots d'Aimé Césaire, « sans qui la terre ne serait pas la terre ${ }^{19}$.»

\section{NOTES}

1. - La mère de Furcy était esclave. Sa propriétaire l'ayant émancipée dans son testament, son fils Furcy était libre, selon l'article du Code Noir qui faisait de l'enfant d'une femme libre une personne libre. Les héritiers ne l'entendirent pas ainsi et gardèrent Furcy dans l'ignorance de son 
statut. Ce dernier découvrit la vérité par hasard. Il porta plainte mais l'affaire fut entravée par les colons réunionnais et la timidité des juges. Ce ne fut que 27 ans jour pour jour après le dépôt de sa plainte que Furcy fut reconnu libre. AISSAOUI, Mohammed. L'Affaire de l'esclave Furcy. Paris : Gallimard, 2010.

2. - Textes disponibles sur www.cpmhe.fr.

3. - Le projet proposait de mettre en scène l'histoire du peuplement de La Réunion à travers les croisements de population dans l'océan Indien, espace millénaire de rencontres et d'échanges. Pour mieux comprendre cette histoire, le «temps » était celui de l'océan Indien afin de montrer les mondes d'où vinrent colons, esclaves, engagés, migrants... L'espace était celui du croisement de «six mondes" - malgache, africain, français et européen, musulman, hindou et chinois. Partant de l'observation que peu d'objets matériels subsistent qui témoigneraient de la vie des esclaves, des colons, des travailleurs engagés, des migrants non-européens (Gujerati, Chinois, Hindous...), la notion d'un «musée sans objets » fut développée, c'est-à-dire imaginer une scénographie qui ne se focalise pas sur l'objet mais mette en scène l'évocation d'un itinéraire, à travers une installation où se mêlent sons, images mouvantes et fixes, archives, objets reconstitués... Le projet a été arrêté en avril 2010.

4. - KOPITOFF, Igor, 1986. "The cultural biography of things: commoditization as process". Dans APPADURAI, Arjun (ed.). The Social Life of Things: Commodities in cultural perspective. Cambridge: Cambridge University Press, 1986, p. 64-91.

5. - VERGÈS, Françoise et MARIMOUTOU, Carpanin. Amarres. Créolisatons india-océanes. Paris : L'Harmattan, 2004, p.

6. - LARSON, Pier M. Ocean of Letters. Language and Creolization in an Indian Ocean Diaspora. Cambridge : Cambridge University Press, 2009.

7. - GUÈYE, Mbaye. «La tradition orale dans le domaine de la traite négrière ». Dans Tradition orale et archives de la traite négrière. Paris : UNESCO, 2001, p. 16-17.

8. - GUÈYE, Mbaye. "La tradition orale dans le domaine de la traite négrière ». Dans Tradition orale et archives de la traite négrière. Paris : UNESCO, 2001, p. 21.

9. - BARRY, Ismael. «Le Fuuta-Jaloo (Guinée) et la traite négrière atlantique dans les traditions orales ». Dans Tradition orale et archives de la traite négrière. Paris : UNESCO, 2003, p. 59.

10. - CARNEY, Judith A., ROSOMOFF, Richard Nicolas. In the Shadow of Slavery. Africa's Botanical Legacy in the Atlantic World. Berkeley: University of California Press, 2009, p. 59.

11. - CARNEY, Judith A., ROSOMOFF, Richard Nicolas. In the Shadow of Slavery. Africa's Botanical Legacy in the Atlantic World. Berkeley: University of California Press, 2009, p. 128.

12. - Voir les remarques dans ÈVE, Prosper. L'Amour à Bourbon au temps de l'esclavage. Saint-Denis, Réunion : Océan éditions, 1998.

13. - FANON, Frantz. Pour la Révolution africaine. Paris : Maspero, 1964, p. 184.

14. - GOODY, Jack. Le Vol de l'histoire. Comment l'Europe a imposé le récit de son passé au reste du monde . Paris : Gallimard, 2010.

15. - GOODY, Jack. Le Vol de l'histoire. Comment l'Europe a imposé le récit de son passé au reste du monde . Paris : Gallimard, 2010, p. 13.

16. - THIOUB, Ibrahima. "Traites et esclavages en Afrique». Dans COTTIAS, Myriam, CUNIN, Elisabeth, et de ALMEIDA MENDES, António. Les traites et les esclavages: Perspectives historiques et contemporaines. Karthala, 2011, p. 377-386.

17. - Voir l'ouvrage de : COQUERY-VIDROVITCH, Catherine. Une Histoire de l'Afrique en abrégé. Paris : La Découverte, 2011.

18. - «Tabous de l'esclavage. Entretien avec Ibrahima Thioub». Entretien réalisé par Camille BAUER. L'Humanité, 24 juin 2008.

19. - CÉSAIRE, Aimé. Cahier d'un retour au pays natal. Paris : Présence Africaine, 2000. 


\section{RÉSUMÉS}

Depuis quelques années, un nouveau regard est jeté sur les mémoires et les patrimoines de la traite négrière et de l'esclavage. Ce renouveau a été impulsé par le mouvement social et culturel qui a émergé en 1998 lors du $150^{\mathrm{e}}$ anniversaire de l'abolition de l'esclavage dans les colonies françaises et a été renforcé en 2001 par l'adoption de la loi reconnaissant la traite négrière et l'esclavage comme "crime contre l'humanité». Dans cette contribution, Françoise Vergès souligne l'importance de ce tournant. Elle revient sur la culture immatérielle, centrale, pour évoquer l'expérience des esclaves. La mémoire de la traite négrière et de l'esclavage est donc devenue une " pratique sociale ». Le terrain n'est pas neutre et c'est pour cela que rendre le plus large possible l'accès aux archives et autres patrimoines, en l'accompagnant de programmes éducatifs est une tâche essentielle.

In France, the memories of slave trade and slavery have been reactivated since 1998 when the country celebrated the $150^{\text {th }}$ anniversary of the abolition of slavery. A law was voted in May 2001 which recognized slave trade and slavery as « crime against humanity » and set up a Committee whose task was to elaborate propositions in the fields of school, research and culture to further the knowledge of this history. A cultural and social movement has emerged in the ten years since the adoption of the law. In this contribution, Françoise Vergès argued that this movement has opened up new questions and led to a new methodology of reading the archives and looking at findings in archeology and at the ways in which museums on slavery are thought. Special attention must be paid to intangible culture which constitutes an important source created by the enslaved.

INDEX

Mots-clés : mémoire, culture immatérielle, genre, cartographies

\section{AUTEUR}

\section{FRANÇOISE VERGÈS}

Présidente du Comité pour la mémoire et l'histoire de l'esclavage vergesf@free.fr 